\title{
. The impact of the Kasatochi eruption on the Moon's illumination during the August 2008 lunar eclipse
}
A. García Muñoz, ${ }^{1,2}$ E. Pallé, ${ }^{1,2}$ M.R. Zapatero Osorio, ${ }^{3}$ and E.L. Martín ${ }^{3}$

\author{
${ }^{1}$ Instituto de Astrofísica de Canarias \\ (IAC), C/ Vía Láctea s/n, E-38200 La \\ Laguna, Tenerife, Spain \\ ${ }^{2}$ Departamento de Astrofísica, \\ Universidad de La Laguna (ULL), E-38206 \\ La Laguna, Tenerife, Spain \\ ${ }^{3}$ Centro de Astrobiología, CSIC-INTA, \\ Madrid, Spain
}


3 The Moon's changeable aspect during a lunar eclipse is largely attributable ${ }_{4}$ to variations in the refracted unscattered sunlight absorbed by the terres${ }_{5}$ trial atmosphere that occur as the satellite crosses the Earth's shadow. The - contribution to the Moon's aspect from sunlight scattered at the Earth's ter7 minator is generally deemed minor. However, our analysis of a published spec\& trum of the 16 August 2008 lunar eclipse shows that diffuse sunlight is a ma9 jor component of the measured spectrum at wavelengths shorter than 600 ${ }_{10} \mathrm{~nm}$. The conclusion is supported by two distinct features, namely the spectrum's tail at short wavelengths and the unequal absorption by an oxygen

12

collisional complex at two nearby bands. Our findings are consistent with the presence of the volcanic cloud reported at high northern latitudes following the 7-8 August 2008 eruption in Alaska of the Kasatochi volcano. The cloud both attenuates the unscattered sunlight and enhances moderately the scattered component, thus modifying the contrast between the two contributions. 


\section{Introduction}

The classical theory of lunar eclipses accounts for refraction, differential absorption and focusing to explain the Moon's aspect during an eclipse [Link, 1962]. Link's classical theory has been subsequently perfected and used to investigate the composition and aerosol loading of the Earth's atmosphere [e.g., Ugolnikov and Maslov, 2008]. Aerosols play a critical role in the interpretation of lunar eclipses as their content, distribution and optical properties are largely unpredictable. Volcanic eruptions and meteor showers are two natural sources of aerosols with the potential for perturbing the atmosphere and, in turn, the aspect of the eclipsed Moon [Keen, 1983; Vollmer and Gedzelman, 2008]. Occasionally, large wildfires may also perturb the atmosphere [Fromm et al., 2010].

García Muñoz and Pallé [2011] have revisited the lunar eclipse theory and estimated the impact of volcanic aerosols on the spectrum of sunlight at the eclipsed Moon. Aerosols may substantially attenuate the direct sunlight while simultaneously enhancing somewhat the scattered contribution. The latter depends strongly on the capacity of aerosols for forwardscattering the incident light and, consequently, on the aerosols' size. The spectroscopic characterization of the sunlight reflected from the eclipsed Moon takes the investigation of lunar eclipses farther than allowed for by photometry and the traditional color indices.

The 7-8 August 2008 eruption of the Kasatochi volcano $\left(52.17^{\circ} \mathrm{N}, 175.51^{\circ} \mathrm{W}\right.$, Aleutian Islands, Alaska) ended a period of global low stratospheric aerosol amounts. The three main explosions recorded over two days plus the release of gas that followed for hours delivered into the atmosphere $\sim 1.5 \mathrm{Tg}$ of $\mathrm{SO}_{2}$ [Waythomas et al., 2010], which is $\sim 30$ times less than the $\mathrm{SO}_{2}$ injected by Pinatubo in 1991. The plume of gas and ash rose up 


\section{Data}

47

to $\sim 14-18 \mathrm{~km}$ and drifted eastward carried by jet winds, spreading rapidly over North America, Greenland, and the North Atlantic Ocean. The cloud was spotted above Europe on 15 August, one week after the eruption [Martinsson et al., 2009].

Our paper shows that a published spectrum of the Moon in umbra during the August 2008 lunar eclipse contains sunlight scattered at the Earth's terminator. We argue that the Kasatochi eruption is the most plausible origin for the abnormally elevated atmospheric opacity needed to explain the observation. Vidal-Madjar et al. [2010] have published a spectrum of the August 2008 lunar eclipse, but covering only the penumbra.

We use data of the 16 August 2008 lunar eclipse obtained with the ALFOSC instrument mounted on the Nordic Optical Telescope at the Observatorio del Roque de los Muchachos (La Palma, Spain) and presented by Pallé et al. [2009]. The dataset comprises spectra of the Moon in umbra (21:36UT), penumbra (22:11UT), and out of eclipse (23:09UT). Pallé et al. [2009] derived a lunar eclipse spectrum from the ratio of umbra and penumbra spectra. The ratio cancels out the solar spectrum and the telluric signature of the Moonto-telescope optical path. What remains is the imprint of the limb-viewed terrestrial atmosphere (averaged in a particular way over the terminator) on the sunlight that reaches the Moon in umbra. Our analysis sets out from the 400-900 nm published spectrum.

The solar elevation angle, $e$, is the geocentric angle between the incident sunbeam direction and the direction from the Earth's centre to the lunar disk parcel targeted by the telescope. We have that $e \sim 0.34^{\circ}$ for the slit projected on the Moon. The structure of an umbra spectrum is very sensitive to e [García Muñoz and Pallé, 2011]. 


\section{Evidence of scattered sunlight}

\subsection{The short-wavelength tail of the spectrum}

60 Model predictions for $e \sim 0.34^{\circ}$ and a broad range of aerosol loadings show that the

61

62

63

spectrum of sunlight directly transmitted through the atmosphere is typically $2-3$ orders of magnitude fainter at $400 \mathrm{~nm}$ than at $600 \mathrm{~nm}$ [García Muñoz and Pallé, 2011]. This is at odds with the eclipse data, which show that the measured spectrum is roughly flat to within a factor of 2 shortwards of $600 \mathrm{~nm}$ and that the fluxes at 400 and $880 \mathrm{~nm}$ are in a ratio of $\sim 1: 20$. It thus means that direct sunlight is not the only contributor to the measured spectrum. García Muñoz and Pallé [2011] note that a flat spectrum at short wavelengths indicates that diffuse sunlight dominates locally over direct sunlight.

\subsection{The $\left(\mathrm{O}_{2}\right)_{2}$ bands at 577 and $630 \mathrm{~nm}$}

We fitted synthetic curves of the form $\prod_{i} \exp \left(-\tau_{i}\right)$ to the measured spectrum from 550 to $660 \mathrm{~nm}$. The curves include absorption by $\mathrm{H}_{2} \mathrm{O}, \mathrm{O}_{3}, \mathrm{O}_{2}$ and the $\left(\mathrm{O}_{2}\right)_{2}$ collisional complex. One term, $\tau_{\text {cont }}=\sum_{k=0}^{4} c_{k}\left(\lambda_{*} / \lambda\right)^{k}$, with $\lambda_{*}=600 \mathrm{~nm}$, accounts for a continuum baseline. Thus, each curve contains up to ten degrees of freedom, namely, five $c_{k}$ 's, integrated columns for $\mathrm{H}_{2} \mathrm{O}, \mathrm{O}_{3}$ and $\mathrm{O}_{2}$, and, optionally, one integrated column for each of the $\left[X^{3} \Sigma_{g}^{-}(0)\right]_{2} \rightarrow a^{1} \Delta_{g}(0)+a^{1} \Delta_{g}(1)$ and $\left[X^{3} \Sigma_{g}^{-}(0)\right]_{2} \rightarrow\left[a^{1} \Delta_{g}(0)\right]_{2}$ bands of $\left(\mathrm{O}_{2}\right)_{2}$ that occur at 577 and $630 \mathrm{~nm}$, respectively. For the temperature-dependent gas properties, the temperature was fixed at $225 \mathrm{~K}$. The synthetic curves were properly degraded and resampled. The minimization of $\chi^{2}=\sum_{j}\left(1-I_{\text {fit }}\left(\lambda_{j}\right) / I_{\exp }\left(\lambda_{j}\right)\right)^{2}$, where $I_{\text {fit }}$ and $I_{\exp }$ are the synthetic and observed data, outputs the best fit parameters. 
Figure (1) summarizes the best fits obtained from three separate strategies, each of them treating the 577 and $630 \mathrm{~nm}$ bands of $\left(\mathrm{O}_{2}\right)_{2}$ in a different manner. Strategy A fits the spectrum with null amounts of $\left(\mathrm{O}_{2}\right)_{2}$; B includes $\left(\mathrm{O}_{2}\right)_{2}$ and assumes the same integrated column for the two bands; and $\mathrm{C}$ allows for separate integrated columns for each of the 577 and $630 \mathrm{~nm}$ bands. In the top panel, the solid black curves represent the measured spectrum, shifted in the vertical for comparison with the synthetic curves. The red solid curves are the respective $\mathrm{A}, \mathrm{B}$ and $\mathrm{C}$ best fits. The bottom panel displays the residuals. Including $\left(\mathrm{O}_{2}\right)_{2}$ reduces notably the fit residuals. The fit improves further if the integrated column at $630 \mathrm{~nm}$ is about twice the column at $577 \mathrm{~nm}$. The latter conclusion is the core of the second argument that proves the significance of diffuse sunlight in the measured lunar eclipse spectrum. Some comments on the robustness of the fitting procedure can be found in the Supplementary Material.

Taking $\mathrm{C}$ as the optimal strategy, the conclusion is that average sunlight photons at 577 and $630 \mathrm{~nm}$ follow different paths in the atmosphere. The direct trajectories of sunlight rays are dictated by the atmospheric refractive index, which does not change appreciably within such a narrow spectral interval. The amount of sunlight directly transmitted does however vary sharply with wavelength. Direct sunlight is more attenuated at $577 \mathrm{~nm}$ than at $630 \mathrm{~nm}$ due to the $\sim \lambda^{-4}$ behaviour of the Rayleigh cross section and the closer proximity of the $577 \mathrm{~nm}$ band to the absorption peak of the $\mathrm{O}_{3}$ Chappuis band.

We thus have to invoke sunlight scattered at the Earth's terminator to explain the measured spectrum. García Muñoz and Pallé [2011] show that in a lunar eclipse the bulk of diffuse sunlight near $600 \mathrm{~nm}$ originates from above $15 \mathrm{~km}$. In the stratosphere, 
the $\left(\mathrm{O}_{2}\right)_{2}$ density, which drops with a scale height half that of the background density, is negligibly small. Foreseeably, the signature of the $\left(\mathrm{O}_{2}\right)_{2}$ bands in the diffuse sunlight spectrum is weak.

\section{Analysis and discussion}

Next, we generate model lunar eclipse spectra that reproduce the measured spectrum if a few reasonable assumptions on the loading and properties of airborne aerosols are introduced. The spectra, generated with the model described by García Muñoz and Pallé [2011], contain both direct and diffuse components. Further details on the underlying model assumptions can be found in the Supplementary Material.

The tracing of the direct sunbeam that reached the parcel of the Moon's disk tracked by the telescope reveals that the sunbeam intercepted the volcanic cloud formed in the Kasatochi eruption, as seen in Fig. (2). It is expected that the direct sunlight component is more strongly affected by the volcanic cloud than the diffuse one, which originates from all terminator locations. This distinction is accounted for in the generation of the model spectra by assuming separate atmospheres for the calculation of each component.

For simplicity, the model spectra are allowed only four adjustable parameters. These are $f_{\gamma_{0}}, \alpha^{\prime}$ and $f_{\mathrm{O}_{3}}$ for the direct sunlight component, and $r_{\text {eff }}$ for the diffuse one. In the former, $f_{\gamma_{0}}$ scales the reference aerosol extinction profile at $1.02 \mu \mathrm{m}, \alpha^{\prime}$ is the Ångström exponent to extrapolate the $1.02-\mu \mathrm{m}$ extinction profile to shorter wavelenghts, and $f_{\mathrm{O}_{3}}$ scales the reference ozone profile. In the calculation of the diffuse sunlight component, $r_{\text {eff }}$ stands for a mean effective radius for aerosols at the terminator. The sulfate droplets of background aerosols in the quiescent atmosphere have $r_{\text {eff }} \sim 0.1-0.2 \mu \mathrm{m}$, whereas volcanic 
et al., 2003]. Exponents $\alpha^{\prime} \sim 0-0.15$ are indicative of large-size particles being carried in the volcanic cloud. The conversion from $\mathrm{SO}_{2}$ to sulfate droplets has an e-folding time of 20-50 days [Kristiansen et al., 2010]. It is unlikely that one week after the eruption is enough time for large sulfate droplets to form. Thus, the $\alpha^{\prime}$ values inferred suggest that the volcanic cloud carried sizeable amounts of unsedimented ash. Sioris et al. [2010] report small Ångström exponents of 0.5 in early September 2008.

The inset of Fig. (3) shows the two component spectra near $600 \mathrm{~nm}$. The diffuse spectrum is roughly flat and shows no evidence of $\left(\mathrm{O}_{2}\right)_{2}$ absorption. When the direct and diffuse model spectra are added, the $577 \mathrm{~nm}$ band becomes more diluted than the 630 $\mathrm{nm}$ band, which translates into an effective integrated column at $630 \mathrm{~nm}$ larger than at $577 \mathrm{~nm}$. For the case in Fig. (3) the ratio is $\sim 1: 1.4$, somewhat smaller than the $\sim 1: 2$ ratio inferred from the measured spectrum. One may generally state that comparable amounts of direct and scattered sunlight near $600 \mathrm{~nm}$ lead to larger $\left(\mathrm{O}_{2}\right)_{2}$ columns at the longer-wavelength band.

Figure (I) in the Supplementary Material proves that good fits to the measured spectrum are possible for the full $r_{\text {eff }}$ set. This means that the measured spectrum accepts one quantitative interpretation for each $r_{\text {eff }}$. In qualitative terms, though, the picture that we obtain is fairly consistent and indicates that the direct sunbeam was substantially attenuated by the volcanic cloud, which leads to an enhanced contrast of the diffuse component. For future efforts, we suggest that the flux calibration of the undivided spectra might help break the degeneracy. 
A comment is to be made regarding the Ring effect and the structure seen in the measured spectrum shortwards of $540 \mathrm{~nm}$. The Ring effect refers to the smearing of solar Fraunhofer lines that occurs in the spectrum of sunlight scattered in the atmosphere [Grainger and Ring, 1962]. In the Earth's atmosphere, the Ring effect is due to rotational Raman scattering by $\mathrm{N}_{2}$ and $\mathrm{O}_{2}$ [Kattawar et al., 1981]. Raman scattering redistributes in wavelength part of the incident photons, the redistribution being more evident where the incident solar spectrum shows the sharpest lines. The ratio of scattered to unscattered sunlight spectra reveals the Ring effect as a filling-in of the solar line cores. The detection of the Ring effect in the eclipse data would mean a further confirmation of scattered sunlight. The measured lunar eclipse spectrum shows that ripples do occur in the Fraunhofer region. The sign of the structures is however inverted with respect to what the Ring effect would produce. The inspection of the undivided umbra spectrum shows that the solar lines are unexpectedly deep, probably due to the limitation in the subtraction of the sky spectrum at these wavelengths, where the signal-to-noise ratio is the lowest. Thus, the structure seen in the measured spectrum cannot be attributed to the Ring effect. Further, a few quantitative arguments allow us to deem as minor the impact of the Ring effect on the measured lunar eclipse spectrum. Following Kattawar et al. [1981], the filling-in for forward-scattered sunlight is $\sim 2.5 \%$ of the continuum Rayleigh-scattered by the gas. In the conditions explored here the filling-in would be undetectably small because the sunlight scattered by the gas contributes less than a few percent to the net sunlight scattered by gas and aerosols together. 
Pyrocumulonimbus (pyroCbs) is a recently-coined term to designate convective activity triggered or sustained by wildfires [Fromm et al., 2010]. In extreme events, pyroCbs inject smoke and biomass-burning particles into the troposphere and lower stratosphere and alter the global aerosol loading. PyroCbs may result in aerosol extinctions $\sim 10^{-3}-10^{-2} \mathrm{~km}^{-1}$ well above the tropopause, opacities that are often associated with volcanic clouds. It would be difficult to differentiate the impact on the eclipsed Moon of one such event from that of a volcanic eruption. To our knowledge, no extreme pyroCbs were reported in the weeks preceding the eclipse, a period that was monitored with unprecedented detail. Thus, if any, the contribution in the eclipse of pyroCbs blended with that of the Kasatochi cloud.

The Perseids is one of the most copious meteor showers, running yearly from late July to late August. In 2008, its peak of activity occurred near 13 August. Despite recent work [Mateshvili et al., 1999; Renard et al., 2010], there are significant uncertainties on the optical properties of the atmosphere perturbed by meteor showers. Mateshvili et al. [1999] report two-fold enhancements with respect to pre-shower values in the twilight brightness above $20 \mathrm{~km}$ during the Leonids in 1998. Assuming that both meteor showers are comparable and that the brightness enhancement translates into a similar increase in stratospheric opacity, the effect of extraterrestrial dust would be more than one order of magnitude smaller than that by the volcanic perturbation. Thus, the effect of meteoroid dust in the measured spectrum is likely masked by the volcanic perturbation.

We have shown that the lunar eclipse spectrum published by Pallé et al. [2009] was affected by sunlight scattered at the Earth's terminator. We offered theoretical arguments 
that hint at the Kasatochi eruption as the most plausible origin for the atmospheric perturbation needed to explain the observations. Future observations will allow us to compare lunar eclipse spectra obtained in different atmospheric conditions. In a broader context, it is worth mentioning that the retrieval of globally-averaged atmospheric optical properties is a relevant exercise towards the future characterization of transiting Earthlike extrasolar planets. As a corollary, we may state that the color of the lunar disk in umbra during the 16 August 2008 lunar eclipse was partly caused by diffuse sunlight.

Acknowledgments. ELM acknowledges a Visiting Research Professorship at the Department of Geological Sciences of the University of Florida. We thank the two reviewers for constructive comments.

\section{References}

Bauman, J.J., P. B. Russell, M. A. Geller, and P. Hamill (2003), A stratospheric aerosol climatology from SAGE II and CLAES measurements: 2. Results and comparisons, 1984-1999, J. Geophys. Res., 108, 4383, doi:10.1029/2002JD002993.

Fromm, M., D.T. Lindsey, R. Servranckx, G. Yue, T. Trickl, R. Sica, P. Doucet, S. GodinBeekmann (2010), The untold story of pyrocumulonimbus, BAMS, 91, 1193-1209.

García Muñoz, A., and E. Pallé (2011), Lunar eclipse theory revisited: Scattered sunlight in both the quiescent and the volcanically perturbed atmosphere, JQSRT, 112, 16091621.

Grainger, J.F., and J. Ring (1962), Anomalous Fraunhofer line profiles, Nature, 193, 762. 
Kattawar, G.W., A.T. Young, and T.J. Humphreys (1981), Inelastic scattering in planetary atmospheres. I. The Ring effect, without aerosols, Astrophys. J., 243, 1049-1057.

Keen, R. (1983), Volcanic aerosols and lunar eclipses, Science, 222, 1011-1013.

Kristiansen, N.I., A. Stohl, A.J. Prata, A. Richter, S. Eckhardt, et al. (2010), Remote sensing and inverse transport modeling of the Kasatochi eruption sulfur dioxide cloud, J. Geophys. Res., 115, D00L16, doi:10.1029/2009JD013286.

Link, F. (1962), Lunar eclipses. In: Kopal Z, editor. Physics and Astronomy of the Moon, p. $161-229$.

Martinsson, B.G., C. A. M. Brenninkmeijer, S. A. Carn, M. Hermann, K.-P. Heue, P.F.J. van Velthoven, and A. Zahn (2009), Influence of the 2008 Kasatochi volcanic eruption on sulfurous and carbonaceous aerosol constituents in the lower stratosphere, Geophys. Res. Lett., 36, L12813, doi:10.1029/2009GL038735.

Mateshvili, N., G. Mateshvili, I. Mateshvili, L. Gheondjian, and O. Avsajanishvili (1999), Vertical distribution of dust particles in the Earth's atmosphere during the 1998 Leonids, Meteoritics 83 Planet. Science, 34, 969-973.

Muñoz, O., H. Volten, J.W. Hovenier, B. Veihelmann, W.J. van der Zande, et al. (2004), Scattering matrices of volcanic ash particles of Mount St. Helens, Redoubt, and Mount Spurr volcanoes, J. Geophys. Res., 109, D16201, doi:10.1029/2004JD004684.

Pallé, E., M.R. Zapatero Osorio, R. Barrena, P. Montañés-Rodríguez, and E.L. Martín (2009), Earth's transmission spectrum from lunar eclipse observations, Nature, 459, $814-816$. 
$\mathrm{X}-14$

GARCÍA MUÑOZ ET AL.: VOLCANIC AEROSOLS IN A LUNAR ECLIPSE

247

Renard, J.-B., G. Berthet, V. Salazar, V. Catoire, M. Tagger, et al. (2010), In situ detection of aerosol layers in the middle stratosphere, Geophys. Res. Lett., 37, L20803, doi:10.1029/2010GL044307.

Sioris, C. E., C. D. Boone, P. F. Bernath, J. Zou, C. T. McElroy, and C. A. McLinden (2010), Atmospheric Chemistry Experiment (ACE) observations of aerosol in the upper troposphere and lower stratosphere from the Kasatochi volcanic eruption, J. Geophys. Res., 115, D00L14, doi:10.1029/2009JD013469.

Ugolnikov, O.S., and I.A. Maslov (2008), Altitude and latitude distribution of atmospheric aerosol and water vapor from the narrow-band lunar eclipse photometry, JQSRT, 109, $378-388$.

Vidal-Madjar, A., L. Arnold, D. Ehrenreich, R. Ferlet, A. Lecavelier des Etangs, et al. (2010), The Earth as an extrasolar transiting planet. Earth's atmospheric composition and thickness revealed by lunar eclipse observations, $A \mathscr{E} A$, 523, A57.

Vollmer, M. and S.D. Gedzelman (2008), Simulating irradiance during lunar eclipses: The spherically symmetric case Appl. Opt., 47, H52-H61.

Waythomas, C. F., W. E. Scott, S. G. Prejean, D. J. Schneider, P. Izbekov, and C. J. Nye (2010), The 7-8 August 2008 eruption of Kasatochi volcano, central Aleutian Islands, Alaska, J. Geophys. Res., 115, B00B06, doi:10.1029/2010JB007437. 

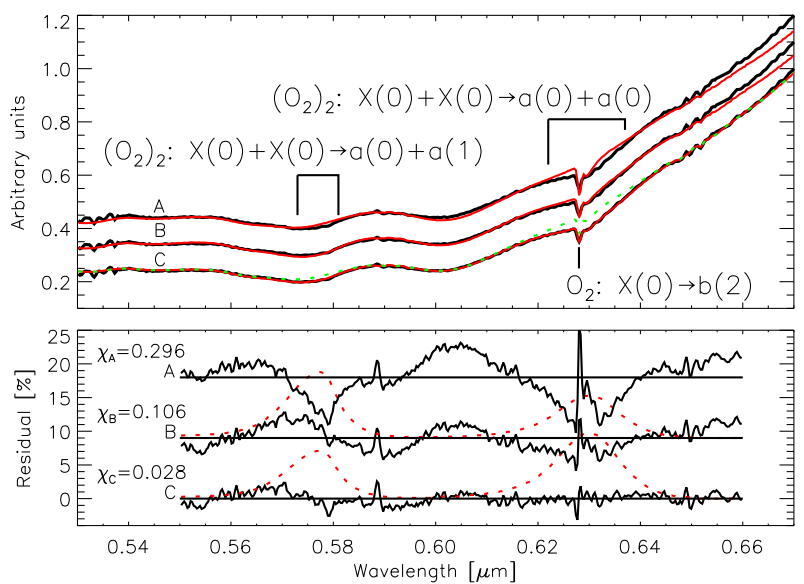

Figure 1. Top: Best fits (red) to the measured spectrum (black) from 550 to $660 \mathrm{~nm}$. The dotted green line are the best fits divided by $\exp \left(-\tau_{\left(\mathrm{O}_{2}\right)_{2}}\right)$. The comparison of the dotted and solid curves makes explicit the contributions from the coincidental in position, albeit distinct in nature, $\mathrm{O}_{2} X(0) \rightarrow b(2)$ and $\left(\mathrm{O}_{2}\right)_{2} X(0)+X(0) \rightarrow a(0)+a(0)$ absorption bands near $630 \mathrm{~nm}$. Bottom: Fit residuals. For B and $\mathrm{C}$, the dotted red curves are the $\left(\mathrm{O}_{2}\right)_{2}$ contributions. In $\mathrm{C}$, we infer an optimal ratio for the 577:630 nm integrated columns of $\sim 1: 2$. 


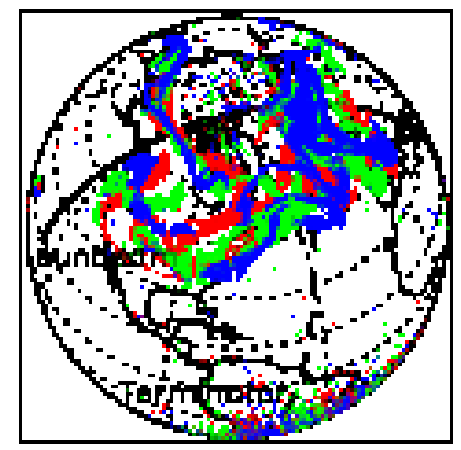

Figure 2. Solid: Projected mid-section trajectory of the sunbeam that reaches the lunar disk targeted by the telescope at 21:36UT on 16 August 2008. Overplotted, the $\mathrm{SO}_{2}$ cloud (a usual volcanic cloud tracer) on 15, 16 and 17 August (red, green and blue, respectively) according to AURA/OMI data (downloaded from the Giovanni online data system, developed and maintained by the NASA GES DISC). The sunbeam's closest approach to the Earth's surface occurs in the North Atlantic region. The local tropopause is at $\sim 10 \mathrm{~km}$. Dashed: Line of the terminator.

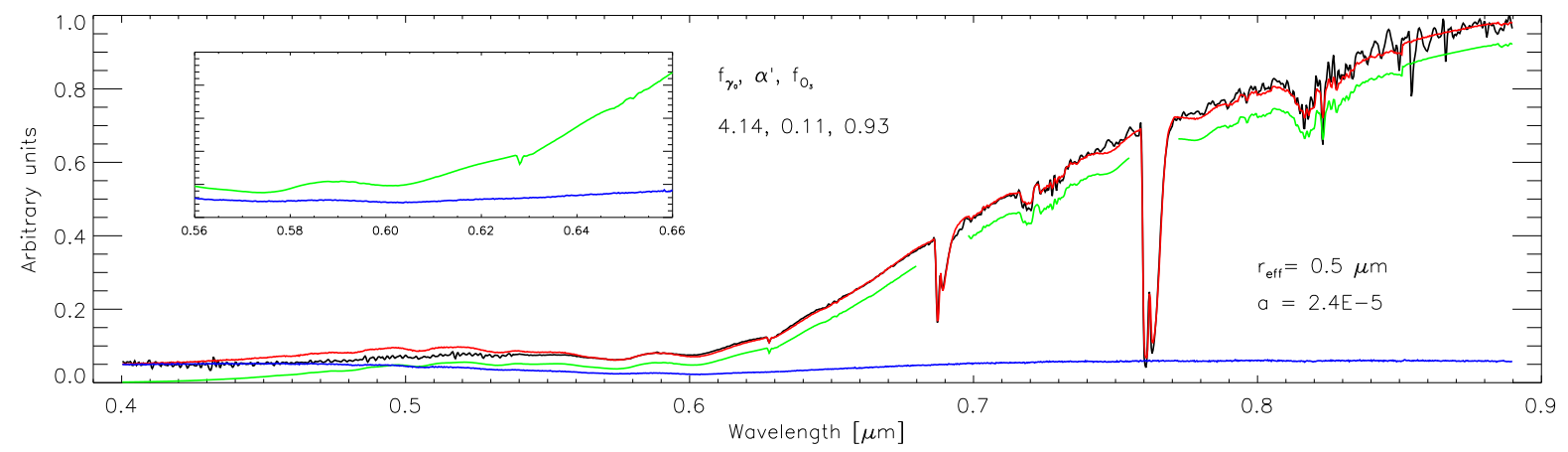

Figure 3. Best model fit for $r_{\text {eff }}=0.5 \mu \mathrm{m}$ (red) to the measured spectrum (black). The model spectrum contains contributions from direct sunlight (green) and diffuse sunlight (blue). The inset is a zoom of the region near $600 \mathrm{~nm}$. The algorithm aims the fit of the continuum away from $\mathrm{O}_{2}$ and $\mathrm{H}_{2} \mathrm{O}$ bands. The $\mathrm{H}_{2} \mathrm{O}$ bands were fitted separately after the fit to the continuum. Figure (I) in the Supplementary Material shows the best fits for the full set of $r_{\text {eff }}$ values. 


\section{Supplementary material}

\section{Additional comments on the robustness of the fitting procedure of Section 3}

Our fitting algorithm uses the laboratory measurements for $\left(\mathrm{O}_{2}\right)_{2}$ cross sections by Greenblatt et al. (1990). Later experiments [Newnham and Ballard, 1998; Naus and Ubachs, 1999] and transmitted moonlight measurements [Wagner et al., 2002] have confirmed that the Greenblatt et al. (1990) cross sections are correct to within a few percent. We verified that using the Newnham and Ballard (1998) cross sections does not modify our conclusion that strategy C performs better than strategies $A$ and $B$. The conclusion remains also unchanged if an alternative temperature in the range 200-250 $\mathrm{K}$ is used to evaluate the temperature-dependent optical properties of the absorbing gases.

\section{Additional information on the spectra simulations of Section 4}

A thorough description of the model used in Section 4 can be found in García Muñoz and Pallé (2011). Vertically-resolved optical properties of the atmosphere are imput to the model. The model also requires the scattering phase functions of all airborne particles. Then, for a selected solar elevation angle, it produces both direct and diffuse spectra of the sunlight amiving at the Moon normalized to the net solar irradiance.

We follow Garcia Muñoz and Palle (2011) for the temperature and densities of the spectroscopically active molecules in the spectral region of investigation (mainly $\mathrm{H}_{2} \mathrm{O}, \mathrm{O}_{2}$ and $\mathrm{O}_{3}$ ). The composite global map of cloud top heights retrieved from GOME-2 data (ICSU World Data Center for Remote Sensing of the Atmosphere, http://wdc.dlr.de/) shows for 16 August 2008 clouds with top heights between 4 and $10 \mathrm{~km}$ at $50-70 \% \mathrm{~N}$ latitudes. In the direct sunlight calculation, we assume that clouds block the sunlight below $6 \mathrm{~km}$. A precise choice of the cloud top height is not critical because for elevated aerosol loadings the atmosphere at that altitude is optically thick in limb viewing. Most of the diffuse sunlight is scattered from higher altitudes, and the choice of the cloud top height in the calculation of the diffuse component is less important.

Several works have investigated the optical properties of the post-eruption atmosphere [e.g., Bourassa et al., 2010; Hoffmamn et al., 2010]. Sioris et al. (2010) have produced 1.02- $\mu$ m extinction profiles from solar occultation data obtained with the Atmospheric Chemistry Experiment (ACE) Imager. The September 2008 ACE Imager extinction profile peaks at altitudes of $\sim 9$ and $15 \mathrm{~km}$, and has an integrated optical thickness above $6 \mathrm{~km}$ of $\sim 0.02$, notably larger than the optical thickness for the same month from 2004 to 2007 . It must be noted that the September 2008 ACE Imager extinction profile (hereafter referred to as $\gamma_{0}\left(\lambda_{0}, z\right)$ ) was infenred from data gathered 2-7 weeks after the eclipse and includes measurements taken over the entire northem hemisphere. Thus, $\gamma_{0}\left(\lambda_{0}, z\right)$ is not directly applicable to the interpretation of the measured lunar eclipse spectrum.

For our direct sunlight calculations, we take as extinction profile by aerosols $\gamma(\lambda, z)=f_{x} \gamma_{0}\left(\lambda_{0}, z\right)$ $\left(\lambda / \lambda_{\omega_{3}}\right)^{\alpha}$, where $\mathrm{f}_{x 0}$ is a mere scaling factor and $\alpha^{\prime}$ is a so-called Angström exponent. Typically, $\alpha^{\prime}$ ranges from 0 , for particles much larger than the incident photon wavelength, to 4 , in the limit of molecule-sized particles. We also allow for ozone extinction in the $400-850 \mathrm{~nm}$ region of the Chappuis band. For this, we take the reference ozone profile of García Muñoz and Pallé (2011) and scale it by an additional parameter $f_{03}$. A battery of $17 \times 5 \times 5=425$ direct sunlight spectra was created to sample the $f_{00}-\alpha^{\prime}-f_{03}$ parameter space in the $[0,8]-[0,2]-[0,2]$ intervals. These spectra are mainly intended to reproduce the contimuum of the measured spectrum away from molecular bands.

As a check, it is worth noting that the values retrieved for $f_{03}$ in the fits of the Section 4 range from 0.85 to 0.95 , which mean (for a reference profile of $\sim 360$ Dobson Units of vertically-integrated ozone) ozone columns between 306 and 342 DU. The total ozone map for 16 August 2008 (World Ozone and Ultraviolet Radiation Data Centre, WOUDC, http://www.woudc.org) shows that in the North Atlantic region near Greenland, where the sumbeam intercepts the terminator, the ozone 
column is $300-350$ DU. Thus, the ozone columns reported at WOUDC and our own retrieved columns are in reasonable agreement.

Our diffuse sunlight calculations use the September 2007 ACE Imager extinction profile as representative of background aerosols. The profile is somewhat arbitranily multiplied by 1.5 to account for some post-eruption global aerosol enhancement. Shortwards of $1.02 \mu \mathrm{m}$, we infer the extinctions by means of an Ångström-type law with an exponent of 1 appropriate to a weakly-tomoderately perturbed atmosphere [Hayashida and Honkawa, 2001]. The total ozone map for 16 August 2008 shows that at the Earth's terminator the average ozone column was less than in our reference profile. Thus, for the diffuse sunlight calculations we have re-scaled the reference profile to a total ozone column of $\sim 250$ Dobson Units.

The phase function of airborne particles at the small scattering angles that occur for forward-, single-scattered sunlight photons is critical in the diffuse problem Multiple processes, which include sedimentation, advection, condensation and coagulation, govem the aerosols' evolving size distribution in the weeks and months after an eruption. It is not known a prion the average global value of $r_{\text {eff }}$ on the date of the eclipse. Thus, we decided to explore the set of $r_{\text {eff }}$ values: $0.1,0.2,0.5$, 1.0 and $2.0 \mu \mathrm{m}$. A size of $\mathrm{r}_{\mathrm{cr}}-0.1 \mu \mathrm{m}$ is representative of an unperturbed atmosphere, whereas $\mathrm{r}_{\mathrm{m}} \sim 2$ $\mu \mathrm{m}$ would mean that the entire terminator is rich in fresh ash In going from $\mathrm{r}_{\text {eff }}=0.1$ to $2 \mu \mathrm{m}$, the phase function in the forward direction (calculated from Mie theory for a unimodal log-normal size distribution, a geometric standard deviation of 2 and a refractive index of 1.45 , M. Mishchenko, www.giss.nasa.gov/staff/mmishchenko/t_matrix.html) augments by $\sim 20$. The phase functions are calculated at a few wavelengths and interpolated in between. 


\section{Bibliography for the Supplementary Material}

Bourassa, A. E., D. A. Degenstein, B. J. Elash, and E. J. Llewellyn (2010), Evolution of the stratospheric aerosol enhancement following the eruptions of Okmok and Kasatochi: Odin OSIRIS measurements, J. Geophys. Res., 115, D00L03, doi:10.1029/2009JD013274.

Garcia Muñoz, A, and E. Pallé (2011), Lunar eclipse theory revisited: Scattered sunlight in both the quiescent and the volcanically perturbed atmosphere, JQSRT, in press.

Greenblatt, G.D., J.J. Orlando, J.B. Burkholder, and A.R Ravishankara (1990), Absorption measurements of oxygen between 330 and $1140 \mathrm{~nm}$, J. Geophys. Res., 95, $18577-18582$.

Hayashida, S., and M Horikawa (2001), Anti-correlation between stratospheric aerosol extinction and the Angstrōm parameter from multiple wavelength measurements with SAGE II - a characteristic of the decay period following major volcanic enutions, Geophys. Res. Lett., 28, $4063-4066$.

Hoffmann, A., C. Ritter, M. Stock, M. Maturilli, S. Eckhardt, et al. (2010), Lidar measurements of the Kasatochi aerosol plume in August and September 2008 in Ny-Ålesund, Spitsbergen, J. Geophys. Res., 115, doi:10.1029/2009JD013039.

Naus, H., and W. Ubachs (1999), Visible absorption bands of the $\left(\mathrm{O}_{2}\right)_{2}$ collision complex at pressures below $760 \mathrm{Ton}$, Appl. Optics, 38, 3,423-3,428.

Newnham, D.A., and J. Ballard (1998), Visible absorption cross sections and integrated absorption intensities of molcular oxygen $\left(\mathrm{O}_{2}\right.$ and $\left.\mathrm{O}_{4}\right)$, J. Geophys. Res., 103, 28,801-28,816.

Sioris, C. E., C. D. Boone, P. F. Bemath, J. Zou, C. T. McElroy, and C. A. McL inden (2010), Atmospheric Chemistry Experiment (ACE) observations of aerosol in the upper troposphere and lower stratosphere from the Kasatochi volcanic eruption, J. Geophys. Res., 115, D00L14, doi: $10.1029 / 2009 \mathrm{JD} 013469$.

Wagner, T., C. von Friedeburg, M. Wenig, C. Otten, and U. Platt (2002), UV-visible observations of atmospheric $\mathrm{O}_{4}$ absorptions using direct moonlight and zenith-sky sunlight for clear-sky and cloudy sky conditions, J. Geophys. Res., 107, 4424, doi:10.1029/2001JD001026. 
Figure mentioned in the main text.

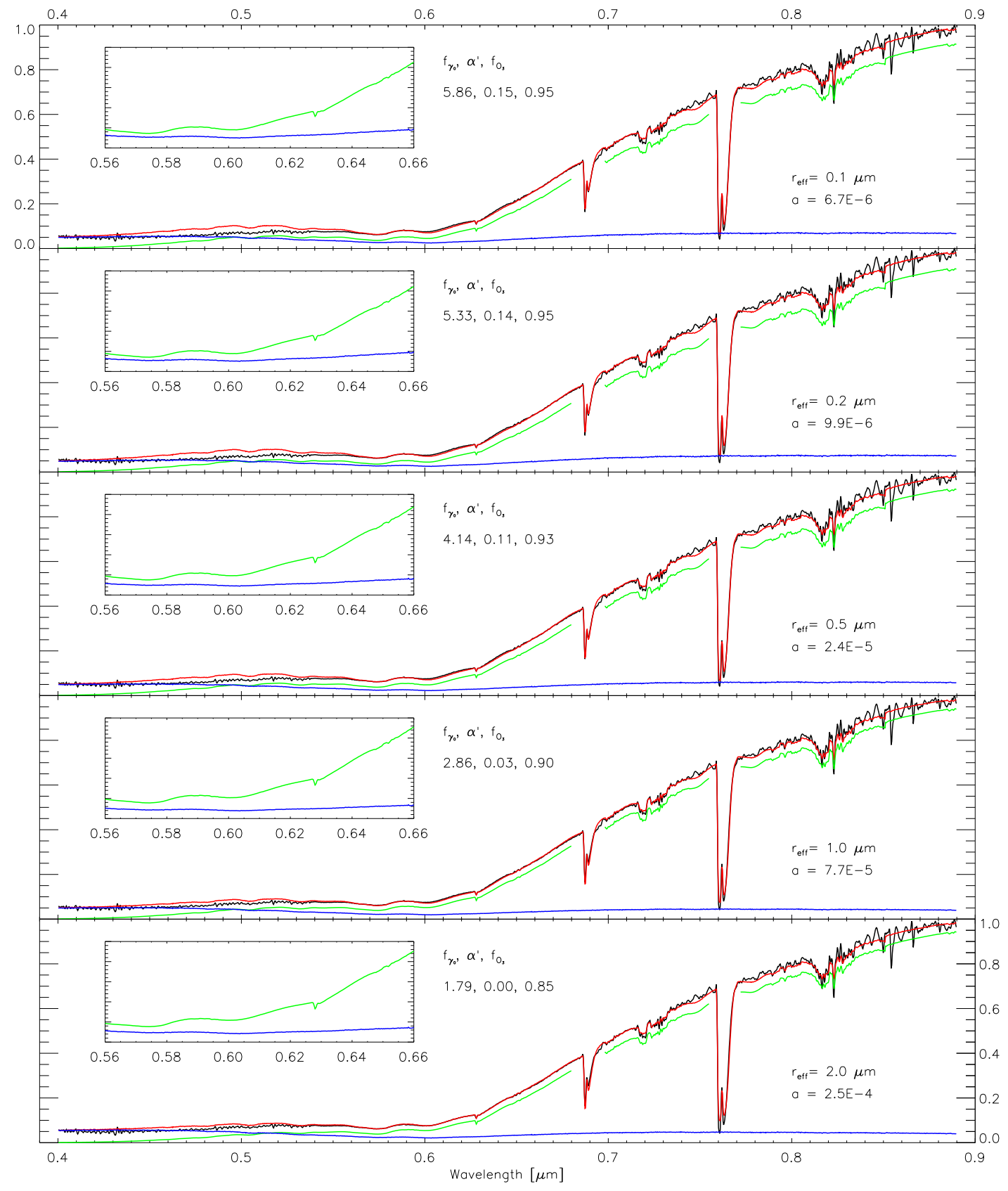

